\title{
Abordando los retos ambientales del gas de pizarra y la fracturación hidráulica
}

\author{
Lucas Vadillo Fernández, Virginia Rodríguez Gómez y Francisco Javier Fernández Naranjo \\ Instituto Geológico y Minero de España. C/Ríos Rosas, 23. 28003 Madrid, España \\ I.vadillo@igme.es; v.rodriguez@igme.es; fj.fernandez@igme.es
}

\begin{abstract}
RESUMEN
El artículo revisa la problemática ambiental del gas no convencional extraído mediante técnicas de fracturación hidráulica. Se exponen aspectos como la tecnología utilizada, las fases de la fracturación hidráulica, las características del agua de retorno y su eliminación, el consumo de agua necesario, las características físico-químicas de las formaciones, el desarrollo de las fracturas realizadas por la fracturación hidráulica, la declinación de los caudales de los pozos, la ocupación del suelo, la sismicidad inducida asociada a la fracturación hidráulica, así como el debate científico abierto acerca de los potenciales pasos del gas metano y la contaminación de acuíferos. Como conclusión se exponen los principales retos ambientales en el caso de España.
\end{abstract}

Palabras clave: Fracturación hidráulica, fugas de gas metano, gas no convencional, ocupación del suelo

\section{Addresing environmental challenges to shale gas and hydraulic fracturing}

\begin{abstract}
This article reviews the main issues of unconventional gas extracted by hydraulic fracturing techniques. Topics such as technology, fracturing stages, flowback characterization and alternatives of disposal and reuse, water consumption, physicochemical features of the geological formations, development of the fractures performed by hydraulic fracturing, well flow decline, land use and occupation and induced seismicity are presented, as well as the scientific debate: the potential steps of methane gas and groundwater contamination.
\end{abstract}

Keywords: hydraulic fracturing, land occupation, methane leakage, unconventional gas

\section{ABRIDGED ENGLISH VERSION}

\section{Introduction}

Hydraulic fracturing and horizontal drilling technologies have developed rapidly reaching a sophisticated process of stimulation of unconventional gas (shale gas and tight gas). Nevertheless, the high number of perforations has resulted in numerous environmental incidents. The bibliographical references of environmental issues such as pollution of aquifers, air pollution and micro-earthquakes are numerous. The interest from oil and gas companies has increased in Spain since considerable resources are expected. More than 70 investigation permits have been approved in five years (Consejo Superior de Colegios de Ingenieros de Minas, 2013).

\section{Shale gas plays}

Shale gas is found in sedimentary formations rich in organic matter, with average porosity in the range of 3 to $6 \%$ and low permeability (0.01 to 0.00007 milidarcys), capable of generating and retaining gas (Bruner and Smosna, 2011). Organic matter, subjected to high pressure and temperature, undergoes physical, chemical and biological transformations. Sediments are naturally distilled with increasing temperature in accordance 
with the depth. Organic matter is transformed into kerogen, precursor for methane gas (Boyer et al., 2006). Shale formations with potential for wet gas in Spain are mainly located in the Basque-Cantabrian Basin, and are considered to contain kerogen type I/II (EIA/ARI, 2013).

\section{Hydrocarbon generation potential of a source rock}

The hydrocarbon generation potential of a source rock is examined through: analysis of total organic carbon content (TOC), an important index to evaluate organic matter abundance (Table 1), the Rock Eval Test, used to identify the type and maturity of organic matter (Table 2) and Vitrinite Reflectance (Ro), used as an indicator of the level of organic maturity (Table 3). Shale formations with potential in Spain are mainly located in the Basque-Cantabrian Basin, and are considered to show a TOC value around 3.0\% and a Ro value of $1.15 \%$ (EIA/ARI, 2013).

\section{Horizontal drilling and fracking}

The fractures are performed by means of the injection of water, sand and chemical additives in the following proportions: $98-99.5 \%$ of water and $0.5-2 \%$ of chemical additives (U.S. Department of Energy, 2009). Approximately $9.5 \%$ of the water composition corresponds to sands that serve to support the newly opened fractures (propant). A drilling and hydraulic fracturing scheme and information about perforation clusters and stage spacing are respectively shown in Figures 1 and 2.

\section{Water consumption}

Water consumption values are very variable and depend on the characteristics of each formation, the vertical depth, and the longitudinal development of the well. These volumes range approximately between 10000 and $16000 \mathrm{~m}^{3}$ per well (U.S. Department of Energy, 2009), reaching $30000 \mathrm{~m}^{3}$ as maximum (New York State Department of Environmental Conservation, 2011). The average of water consumption per well is $10200 \mathrm{~m}^{3}$ in the Barnett Shale field, $11500 \mathrm{~m}^{3}$ in the Fayetteville Shale, $14000 \mathrm{~m}^{3}$ in Haynesville Shale and $14700 \mathrm{~m}^{3}$ in Marcellus Shale (U.S. Department of Energy, 2009).

\section{Chemical and biological components associated with the geological formation}

Chemical and biological components appear in the geological formations as a result of the organic matter transformation. These include: inorganic elements (some of them are radioactivity producers), organic compounds associated with methane (Table 4) and bacteria.

\section{Additives used in hydraulic fracturing}

There is a great diversity of chemical components used for hydraulic fracturing depending on the specific function of each compound in the different phases of the process. The additives can act as: acids (helps dissolve minerals in the water and initiate cracks in the rocks), clay stabilizers (reducing swelling and migration of clays, maintaining thereby the porosity created in fracturing), bactericides/biocides (preventing the growth of bacteria that produce $\mathrm{SH}_{2}$ ), $\mathrm{pH}$ buffers, corrosion inhibitors, crosslinkers (maintaining the viscosity of the fluid when the temperature increases), viscosity reducers (minimizing head loss during pumping), iron controllers (preventing the precipitation of iron dissolved in the form of hydroxides, carbonates), solvents (soluble additives used to control the wettability) and surfactants (reducing surface tension, allowing more free water displacement) (https://fracfocus.org/chemical-use/what-chemicals-are-used).

\section{Flowback}

The volume of the returned water varies greatly depending on the project and the operators. An estimation provided by the UK Environment Agency establishes, as a guideline, that a quarter of the injected water returns to the surface for weeks, even for some months (Environmental Agency U.K, 2011). According to the U.S. Environmental Agency (E.P.A., 2011), returned water varies between 25-75\%. Different concentrations of major elements present in the returned water in Marcellus Shale are presented in Table 5. 
Vadillo Fernández, L., et al., 2016. Abordando los retos ambientales del gas de pizarra... Boletín Geológico y Minero, 127 (4): $775-789$

\section{Potential groundwater contamination}

The radial extent of the fractures depends on the time required for injected fluid pressure to break the rock. The required water pressure and the duration of continuous injection depend on some characteristics of the reservoir, such as lithological variations, stress state, depth, etc. The more water volume injected and the faster the injection, the higher the pressures reached (the Royal Society and the Royal Academy of Engineering, 2012). Osborn et al. (2011) suggest that methane contamination found in aquifers in the vicinity of Utica and Marcellus Shale (Pennsylvania) may have occurred through two possible mechanisms: 1) methane passed laterally and vertically through fractures in the casing, or through uncased wells or with impaired casing in abandoned wells and 2) the applied hydraulic fracturing generated some new fractures or enlarged the existing ones, increasing the hydraulic connectivity with the pre-existing fault systems, being this mechanism less probable than the first one. In order to identify the source of the methane in drinking water wells, Osborn et al. (2011) distinguish between deep methane (thermogenic) and surface methane (biogenic) through isotopic deviation of carbon-13 $(\delta 13 C)$. These wells showed an increase in thermogenic methane, exceeding methane solubility in water with risk of methane explosions. The EPA, in the report Assessment of the Potential Impacts of hydraulic fracturing for Oil and Gas on Drinking Water Resources (E.P.A., 2015), conclude that the identified cases where drinking water resources were impacted in U.S. are small relative to the number of hydraulically fractured wells.

\section{Methane recovery}

The recovery factor, in conventional gas reservoirs, ranges between $80-90 \%$, according to Muggeridge et al. (2014) and 50-80\% according to Jahn, et al. (2008). In non-conventional reservoirs the recovery factor varies between $30 \%$ and less than $15 \%$, with a $30 \%$ recovery factor being considered as exceptional (U.S. Energy Information Administration, 2013). Sandrea and Sandrea (2014) considered that the recovery factor in the principal American sites would be: $1.7 \%$ in Haynesville, $6.1 \%$ in Barnett, $11.2 \%$ in Fayetteville and $9.3 \%$ in Marcellus. The recovery factor depends on several variables such as pore size, hydrostatic pressure in the formation, clay content and tectonic complexity. Formations with high clay content tend to deform rather than break, resulting in relatively few induced fractures. The existence of fault displacements limits directional drilling and the presence of compressive faults reduces permeability and flow capacity (U.S. Energy Information Administration, 2013). The low rate of recovery, along with the loss of pressure, causes a decline in production, forcing the operators to constantly drill new wells in the U.S.

\section{Land occupation}

According to the U.S. Department of Energy (U.S. Department of Energy, 2009) the area occupied by each platform (well pad) is approximately 16000-20000 $\mathrm{m}^{2}$, slightly increased by each well added (multi-well pad). A platform of six to eight wells could occupy an area of $30000 \mathrm{~m}^{2}$ (New York State Department of Environmental Conservation, 2011). The density of wells varies between sites, thus, in the Barnett Shale Play (Texas) drilling density is 5.5 wells per square mile, in Haynesville Shale Play (Louisiana), Fayetteville Shale Play (Arkansas) and in the Marcellus Shale Play (Pennsylvania, New York, Ohio, etc.) the density is 8 wells per square mile (U.S. Energy Information Administration, 2011). This high density of drilling wells is the result of the high declination of their production, forcing operators to continually open new wells.

\section{Induced seismicity}

Some induced seismicity events occurred in shale gas in Bowland (Blackpool, U.K.) and produced enormous media coverage. Fifty earthquakes were detected during hydraulic fracturing operations, from March 31 to May 272011 over an aseismic area. The largest, 2.3 $M_{L}$, occurred on April 1, when the pressure at the bottom of the drilling well was approximately 9900 psi, 68.2 MPa (Eisner et al., 2011), being the pressure needed to open the fractures in the slate. In order to prevent induced seismicity the Royal Society and the Royal Academy of Engineering (2012) proposed a traffic-light system for the monitoring of seismic activity in real time as an essential mitigation strategy. However, the definition of acceptable threshold values for the cessation and commencement of operations is still required. In the Bowland shale, the value initially proposed to cease operations was 1.7 $M_{L}$ (Pater and Baisch, 2011). 
Vadillo Fernández, L., et al., 2016. Abordando los retos ambientales del gas de pizarra... Boletín Geológico y Minero, 127 (4): $775-789$

\section{Conclusions}

Hydraulic fracturing techniques for methane recovery may lead to an environmental impact that must be considered by the relevant environmental authority. The most important impact would be: 1) the loss of environmental quality at the surface of the geological deposit, 2) the loss of agricultural and livestock uses due to the high number of wells and land occupation, 3) the high consumption of water conflicting with other uses in water-scarce areas, 4) the flowback composition, making it difficult to re-use, 5) the possibility of removing the flowback by injecting it into deep aquifers, the disposal option being another environmental issue, 6) the possibility of methane leakage, either through cement fractures, damaged casing of old wells or through fractures and faults that are intersected by the fractures of hydraulic fracturing.

\section{Introducción}

Las técnicas de perforación dirigida y fracturación hidráulica para la estimulación y producción de gas no convencional comenzaron y se han desarrollado en los EE.UU. La técnica de estimulación del gas de pizarra mediante fracturación hidráulica (fracking) en pozos verticales se desarrolló por primera vez a finales de los años 50 del siglo XX, pero no fue aplicado a gran escala hasta finales de los años 80 en el yacimiento de Barnett Shale (Texas). En el año 1992, se realizó por primera vez la perforación horizontal en este mismo yacimiento. En dos décadas la tecnología de fracturación hidráulica y perforación dirigida se ha desarrollado rápidamente hasta alcanzar un sofisticado proceso de estimulación del gas no convencional (shale gas y tight gas).

Este avance tecnológico no se podría entender sin el aumento generalizado del precio del gas y de la dependencia de los EE.UU. de otros países para el abastecimiento de este recurso. Si en el año 2009 la importación neta de gas había disminuido, gracias a la extracción de gas no convencional, se espera que antes del año 2035 EE.UU., sea autosuficiente y virtual exportador de gas gracias a la producción de gas no convencional.

Sin embargo el alto número de perforaciones, consecuencia de la alta declinación del caudal de los pozos, ha dado lugar a numerosas incidencias ambientales. Las referencias bibliográficas de escenarios ambientales con contaminación de acuíferos por metano, contaminación atmosférica y microsismos son numerosas. Situaciones que han dado lugar en varios países europeos (Francia y Bulgaria) a moratorias temporales de varios años, con el fin de llevar a cabo estudios de investigación de los procesos contaminantes en el medio ambiente y prohibiciones que podrían ser o no definitivas, de acuerdo con el estado de conocimiento del momento.

En España, el interés de las compañías operadoras de gas no convencional es creciente. La expectativa de la existencia de considerables recursos prospectivos se ha traducido en una creciente solicitud de permisos de investigación en nuestro país. Durante los años 2008-2013, entre el estado y las comunidades autónomas fueron otorgados más de 70 permisos de investigación, estando pendientes más de 40 solicitudes, considerándose que más de la mitad de los permisos están orientados a la prospección de recursos no convencionales (Consejo Superior de Colegios de Ingenieros de Minas, 2013). Actualmente, dos proyectos de exploración se encuentran en fase de alegaciones e información pública.

En la página web del Ministerio de Industria se puede consultar el "Mapa de posición de permisos de investigación y concesiones de explotación y almacenamiento subterráneo de hidrocarburos", con fecha de actualización de diciembre de 2013, en la siguiente dirección: http://www6.mityc.es/aplicaciones/energia/hidrocarburos/petroleo/exploracion2013/mapas/inicio.html (MINETUR), si bien a priori no es posible distinguir aquellos destinados al gas no convencional.

El presente artículo recopila los principales problemas ambientales que son necesarios abordar por principio de precaución a la hora de plantear en este tipo de proyectos, dado que en España, en materia de gas no convencional, no existen experiencias basadas en la práctica.

\section{Yacimientos de shale gas}

El gas de pizarra o shale gas se encuentra en aquellas formaciones sedimentarias (lutitas, margas) ricas en materia orgánica capaces de generar y retener el gas con una porosidad media entre 3 y $6 \%$ y una permeabilidad en el rango de 0.01 a 0.0007 milidarcis (Bruner and Smosna, 2011).

La materia orgánica una vez sometida a procesos de presión y temperatura sufre una serie de transformaciones físicas, químicas y biológicas. Los sedimentos sufren un proceso de destilación natural a medida que aumenta la temperatura en concordancia con la profundidad de enterramiento. Los sedimentos se litifican y la materia orgánica se transforma en 
Vadillo Fernández, L., et al., 2016. Abordando los retos ambientales del gas de pizarra... Boletín Geológico y Minero, 127 (4): $775-789$

querógeno, precursor del gas metano (Boyer et al., 2006).

El querógeno puede dividirse en cuatro tipos (Boyer et al., 2006; McCarty et al., 2011):

Querógeno Tipo I: generado principalmente en ambientes lacustres, procede de la descomposición de la materia algácea y planctónica, rica en hidrogeno y pobre en oxígeno que genera gas biogénico, aunque también puede producir petróleo. Este tipo de querógeno constituye menos de $3 \%$ de las reservas de petróleo y gas del mundo.

Querógeno Tipo II: se genera en ambientes reductores, en ambientes marinos de profundidad moderada y procede de la descomposición de restos de plancton, rico en hidrógeno y pobre en carbono, pudiendo generar petróleo y gas. Son los yacimientos más abundantes y producen tanto petróleo como gas, pasando progresivamente de petróleo a gas en función de la madurez térmica. Este tipo de gas, denominado gas húmedo (wet gas) contiene metano, etano, propano, n-butano, i-butano y otros hidrocarburos de 5 o más carbonos, e impurezas (mercaptanos, ácido sulfhídrico, nitrógeno, etc.) (Cohe, 2013).

Querógeno Tipo III: procede de la descomposición de restos vegetales terrestres que se han depositado en ambientes marinos y no marinos. Posee menor contenido en hidrogeno y mayor contenido en oxígeno que los anteriores. Este tipo de yacimientos tiende a generar gas seco (dry gas), en la práctica metano casi puro (Cohe, 2013).

Querógeno Tipo IV: es generado a partir de materia orgánica residual. Es altamente rico en carbono y con ausencia de hidrogeno, no presentando potencial de formación de hidrocarburos.

El gas de estos yacimientos se encuentra en forma de gas adsorbido, adherido a la superficie de los componentes orgánicos y de los minerales arcillosos y en forma de gas libre en los poros de las rocas, dentro de los micro-poros de la matriz y en el sistema de microfracturas (Boyer et al., 2006).

En España, los principales yacimientos potenciales del denominado gas de pizarra se encuentran localizados en la Cuenca Vasco-Cantábrica y en la Cuenca del Ebro, si bien, se considera que únicamente la Cuenca Vasco-Cantábrica presenta potencial de explotación, y tendría un querógeno tipo I/II (EIA/ARI, 2013).

\section{Análisis de las rocas generadoras}

El carbono es un elemento esencial de cualquier compuesto orgánico y, por tanto, un parámetro esencial para evaluar las posibilidades de potencial gasífero es el contenido orgánico total (TOC). La Tabla 1 muestra el contenido orgánico total de los principales yacimientos de EE.UU (E.P.A, 2011).

\begin{tabular}{|l|c|}
\hline \multicolumn{1}{|c|}{ Yacimiento shale gas } & TOC (\%) \\
\hline Marcellus & $3-12$ \\
\hline Haynesville & $0.5-4$ \\
\hline Fayetteville & $4-10$ \\
\hline Barnett & 4.5 \\
\hline
\end{tabular}

Tabla 1. Contenido orgánico total (TOC) de los principales yacimientos de EE.UU. Fuente: E.P.A., 2011.

Table 1. Total organic content (TOC) of the main shale plays. U.S.A. (from E.P.A., 2011).

EI TOC indica la cantidad de materia orgánica pero no la calidad. Cuando esta prueba nos dice que existe suficiente materia orgánica, hay que someterla a otras pruebas adicionales para saber la calidad y madurez de ésta, mediante el ensayo Rock-Eval (Behar et al., 2001).

Este ensayo tiene por objeto analizar los gases emitidos por una muestra sometida a un proceso de pirolisis y posterior oxidación. El ensayo se desarrolla en dos fases:

En la primera fase, la muestra de roca se calienta a $300^{\circ} \mathrm{C}$ en una atmósfera inerte, para después ir incrementando la temperatura a razón de $25^{\circ} \mathrm{C}$ por minuto hasta los $850^{\circ} \mathrm{C}$ aproximadamente. Se mide respectivamente la masa de los hidrocarburos gaseosos emitidos (en microgramos por gramo de muestra), concentrados en tres picos denominados: S1, S2 y S3.

La segunda fase del ensayo se realiza en una atmósfera oxidante y se utiliza el residuo del ensayo anterior pero en un horno distinto, resultando otros dos picos más de gas, el S4 y el S5. Este cuarto pico se corresponde con el $\mathrm{CO}_{2}$ y $\mathrm{CO}$ resultantes de la combustión del carbono orgánico residual presente aún en la muestra, mientras que el quinto pico se corresponde con el $\mathrm{CO}_{2}$ resultado de la descomposición de los carbonatos. En la Tabla 2 se recoge el criterio para determinar la capacidad generadora de gas en función del TOC y de los resultados del ensayo Rock-Eval.

Otro de los parámetros utilizados para valorar la madurez térmica es la Reflectancia a la Vitrinita $\left(R_{0}\right)$. La vitrinita es un maceral formado por la alteración térmica de la lignina y la celulosa de las paredes de las células vegetales. Con el incremento de la temperatura, la vitrinita experimenta reacciones de aromatización que se traducen en un incremento de la reflectancia. La Tabla 3 muestra la relación entre el parámetro $R_{0}$ y el tipo de hidrocarburo que se genera. 


\begin{tabular}{|l|c|c|c|}
\hline \multirow{2}{*}{$\begin{array}{c}\text { Calidad } \\
\text { de la roca } \\
\text { generadora }\end{array}$} & \multirow{2}{*}{$\begin{array}{c}\text { TOC } \\
\text { (\%) }\end{array}$} & \multicolumn{2}{|c|}{ Rock-Eval (pirólisis) } \\
\cline { 3 - 4 } & & S1 (mg g-1) & S2 (mg g $\left.\mathbf{~}^{-1}\right)$ \\
\hline Pobre & $0-0.5$ & $0-0.5$ & $0-2.5$ \\
\hline Regular & $0.5-1$ & $0.5-1$ & $2.5-5$ \\
\hline Buena & $1-4$ & $1-2$ & $5-10$ \\
\hline Muy buena & $2-4$ & $2-4$ & $10-20$ \\
\hline Excelente & $>4$ & $>4$ & $>20$ \\
\hline
\end{tabular}

Tabla 2. Criterio de capacidad generadora de gas en función del Carbono Orgánico Total y el ensayo Rock-Eval (Fuente: Bruner and Smosna, 2011).

Table. 2. Criteria of gas generating capacity based on the Total Organic Carbon and the Rock-Eval test (from Bruner and Smosna, 2011).

\begin{tabular}{|c|l|}
\hline $\mathbf{R}_{\mathbf{o}}(\%)$ & \multicolumn{1}{|c|}{ Hidrocarburos generados } \\
\hline$<0.60$ & Inmaduro/ningún hidrocarburo \\
\hline $0.6<\mathrm{R}_{\mathrm{o}}<1.0$ & Ventana de petróleo \\
\hline $1.0<\mathrm{R}_{\mathrm{o}}-<1.4$ & Gas húmedo y petróleo \\
\hline$>1.4$ & Ventana de Gas seco \\
\hline
\end{tabular}

Tabla 3. Relación entre la reflectancia a la vitrinita $\left(\% R_{o}\right)$ y el tipo de hidrocarburo termogénico generado (Fuente: modificada de Bruner and Smosna, 2011).

Table 3. Relationship between vitrinite-reflectance value $\left(\% R_{\mathrm{o}}\right)$ and type of thermogenic hydrocarbon generated (modified from Bruner and Smosna, 2011).

En el caso de los potenciales yacimientos españoles, que estima que las formaciones de la Cuenca Vasco-Cantábrica presentan, de media, un valor de TOC del $3 \%$ y un valor de $\mathrm{R}_{\mathrm{o}}$ del $1.15 \%$. Mientras, la Cuenca del Ebro presentaría un valor de TOC por debajo del $2 \%$, que es el corte utilizado en la evaluación del potencial de una formación (EIA/ARI, 2013).

\section{Perforación dirigida y fracturación hidráulica}

En los proyectos de fracturación hidráulica los sondeos verticales pueden alcanzar una profundidad aproximada de entre 1000 y $4500 \mathrm{~m}$, de acuerdo con la ventana en la que se forma el gas. Una vez alcanzada la formación geológica que contiene el gas, se realiza el sondeo dirigido en la dirección de la capa en la que se realiza la fracturación hidráulica, pudiendo alcanzar una longitud aproximada de 2000-3000 m (Figura 1).

Realizado el sondeo dirigido, se efectúan, en retroceso, una serie de etapas de fracturación (Frac Zone) hasta completar la longitud del sondeo dirigido realizado en la formación. Estas etapas suelen estar constituidas por entre 4 y 6 fracturas con un desarrollo horizontal de entre 250 y 500 pies $(75-150 \mathrm{~m})$, y con una separación entre cada cluster de entre 50 y 100 pies (15-30 m) (Figura 2). Finalizada la inyección, se habrán formado una serie de fracturas radiales que, según la potencia de la capa, pueden tener de media entre 60-120 metros de desarrollo, por donde fluye el gas (Beard, 2011). Si bien como veremos posteriormente algunas fracturas pueden alcanzar desarrollos mayores.

La realización de las fracturas se realiza mediante la inyección de agua, arena y aditivos químicos en las siguientes proporciones: $98-99.5 \%$ de agua y $0.5-2 \%$ de aditivos químicos (U.S. Department of Energy, 2009). Una proporción de aproximadamente el $9.5 \%$ del fluido de fracturación inyectado corresponde a arenas que sirven para apuntalar las fracturas abiertas.

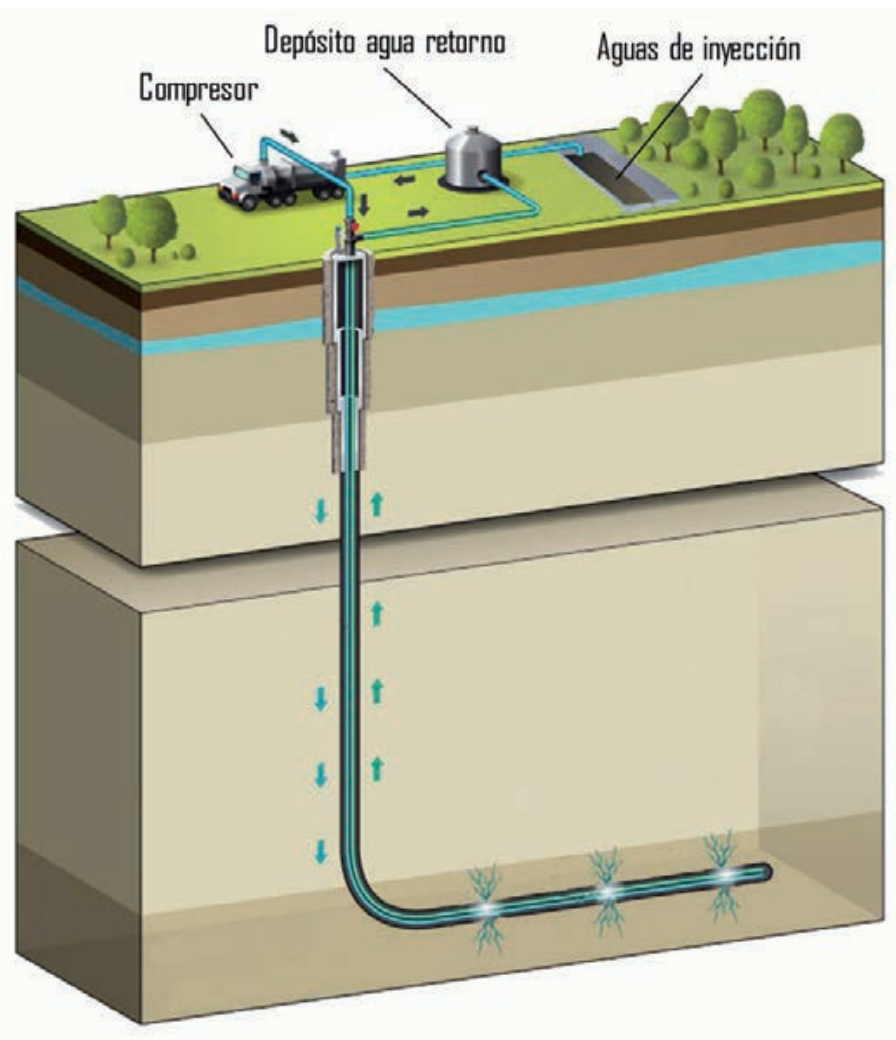

Figura 1. Esquema de sondeos y fracturación hidráulica (modificado de TOTAL: http://www.total.com/en/energies-expertise/oilgas/exploration-production/strategic-sectors/unconventional-gas/ presentation/new-applications-proven-techniques).

Figure 1. Drilling and hydraulic fracturing scheme (modified from TOTAL: http://www.total.com/en/energies-expertise/oil-gas/exploration-production/strategic-sectors/unconventional-gas/presentation/ new-applications-proven-techniques). 


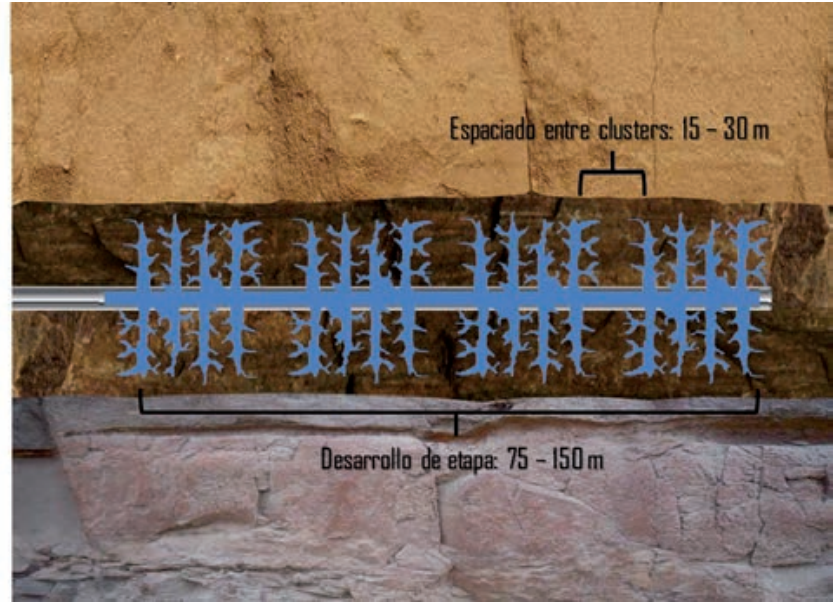

Figura 2. Espaciado entre clusters y desarrollo de etapas (modificado de Beard, 2011).

Figure 2. Perforation cluster and stage spacing (modified from Beard, 2011).

\section{Consumo de agua}

Los valores de consumo de agua necesarios son muy variables y dependen de las características de cada formación, la profundidad vertical y el desarrollo longitudinal del sondeo. Estos volúmenes están comprendidos, aproximadamente, entre 10000 y 16000 $\mathrm{m}^{3}$ (U.S. Department of Energy, 2009), pudiendo alcanzar los $30000 \mathrm{~m}^{3}$ (New York State Department of Environmental Conservation, 2011).

En el yacimiento de Barnett Shale la media de consumo de agua por sondeo es de $10200 \mathrm{~m}^{3}$, correspondiendo $1500 \mathrm{~m}^{3}$ al sondeo vertical; en Fayetteville Shale el consumo es de $11500 \mathrm{~m}^{3}$; en Haynesville Shale es de $14000 \mathrm{~m}^{3}$, donde $3790 \mathrm{~m}^{3}$ corresponden al sondeo vertical; en Marcellus Shale es de $14700 \mathrm{~m}^{3}$ (U.S. Department of Energy, 2009).

Según datos del Comité de Energía y Cambio Climático de la Cámara de los Comunes del Reino Unido sobre Shale Gas, asumiendo una producción del $10 \%$ del gas consumido en el 2008 , se requerirían entre 2500 y 3000 sondeos horizontales sobre una superficie de entre 140 y $400 \mathrm{~km}^{2}$ con unas necesidades de agua de 23 a 86 millones de metros cúbicos de agua (23 a $86 \mathrm{hm}^{3}$ ), 495 plataformas de sondeo (well pad) y un volumen de productos químicos de fracturación de entre 534600 y 1772600 m $^{3}$ (aproximadamente el $2 \%$ de agua inyectada), generándose un volumen de agua de retorno de entre 3920400 y $67953600 \mathrm{~m}^{3}$ (entre el $25 \%$ y el $75 \%$ del agua inyectada (Pickett, 2009; Veil, 2010) y un volumen de productos químicos en el agua de retorno de 78210 a $1359270 \mathrm{~m}^{3}$ (aproximadamente el $2 \%$ de agua de retorno) (House of Commons, 2010-2012).
En el yacimiento de Barnett Shale, uno de los más productivos de EE.UU, el consumo de agua anual que se produjo entre los años 2005-2007, fue de entre 10.3 y 21 millones de metros cúbicos (10.3 a $\left.21 \mathrm{hm}^{3}\right)$. En el 2010, año punta de máximo consumo de agua, fue de 37.7 millones de metros cúbicos $\left(37.7 \mathrm{hm}^{3}\right)$, lo que representa el $1.7 \%$ de la demanda total de agua dulce en el área de Barnett Shale (E.P.A 2011).

\section{Rasgos físico-químicos del medio natural y los fluidos de perforación y fracturación hidráulica.}

Las formaciones geológicas de shale gas contienen elementos y componentes químicos orgánicos, inorgánicos así como bacterias asociados a las pizarras, al gas y a la materia orgánica-gas.

Asimismo, la formación suele estar saturada con aguas congénitas y asociadas a la propia génesis del gas o petróleo, pudiendo llegar a tener concentraciones en solidos disueltos totales (TDS) que varían desde aguas salobres (5000 a 35000 ppm), aguas salinas ( 35000 a 50000 ppm) o salmueras, también denominadas brines (50000 a 200000 ppm); conforme a lo dispuesto por el Instituto Americano del Petróleo (American Petroleum Institute, 2010).

Por tanto, las aguas de retorno en las operaciones de fracturación hidráulica, están constituidas por una mezcla de aguas de la formación, fluidos de perforación $(m u d)$ y los fluidos de fracturación, formados estos últimos por una mezcla de agua ajena al sistema, propantes (arenas, con el objeto de mantener abiertas las fracturas generadas) y aditivos químicos orgánicos e inorgánicos (U.S. Departmet of Energy, 2009; American Petroleum Institute, 2009).

\section{A) Constituyentes del medio natural o fondo geoquímico.}

Los elementos y componentes químicos y biológicos asociados al fondo natural son aquellos que se formaron durante la diagénesis y catagénesis de la materia orgánica hasta su transformación, en parte, en gas. Entre estos componentes y elementos químicos, los hay inorgánicos (elementos metálicos), orgánicos (componentes del gas natural) y seres vivos del Reino Monera (bacterias sulfato reductoras), que se detallan a continuación.

\section{Constituyentes inorgánicos.}

Los constituyentes inorgánicos están asociados a la materia orgánica y a los presentes en las pizarras 
de la formación geológica que, al ser fracturada mediante agua a presión, podrían pasar en disolución al agua residual que se queda en la formación y al agua de retorno del sondeo. Entre dichos elementos químicos, algunos muestran una mayor correlación con la materia orgánica, otros con los sulfuros y en otros casos la correlación no está tan clara ni definida (Leventhal et al., 1981).

Entre los elementos químicos asociados a la materia orgánica y a los sulfuros, encontramos: uranio $(\mathrm{U})$, radio (Ra), molibdeno (Mo), cobalto (Co), níquel $(\mathrm{Ni})$, vanadio $(\mathrm{V})$, mercurio $(\mathrm{Hg})$, arsénico $(\mathrm{As})$, cobre $(\mathrm{Cu})$, plomo $(\mathrm{Pb})$, cinc $(\mathrm{Zn})$, cromo $(\mathrm{Cr})$, antimonio, $(\mathrm{Sb})$, torio (Th), etc. Otros elementos como el magnesio (Mg), calcio (Ca), aluminio (Al), silicio (Si), etc., corresponden a la composición de lutitas, margas, etc.

La disponibilidad de estos elementos químicos en el agua depende del contenido total de metales asociados a las pizarras y a la materia orgánica, así como a la especiación química de cada elemento en función de la relación $\mathrm{pH}$-Eh. Su toxicidad depende, en todo caso, de la concentración de cada elemento en el agua residual y de retorno, que a su vez depende principalmente del $\mathrm{pH}$ y del contenido total de metales en la formación.

Entre los componentes inorgánicos en forma de gas, se encuentra el ácido sulfhídrico $\left(\mathrm{SH}_{2}\right)$, componente habitual en las formaciones anaerobias. El ácido sulfhídrico es un gas más pesado que el aire, incoloro, con el característico olor a huevos podridos, es ligeramente soluble en agua y actúa como un ácido débil, acidificando el medio. Para evitar su toxicidad en superficie, se inyectan biocidas que eliminan las bacterias que reducen los sulfatos a ácido sulfhídrico.

Algunos de los elementos anteriormente descritos, como el uranio y el torio, son radiactivos. Su núcleo tiende a desintegrarse emitiendo radioactividad y dando lugar a otros elementos radiactivos hasta la aparición de un núcleo estable (en este caso el plomo). Entre los elementos radiactivos de trasmutación del uranio podemos encontrar: $\mathrm{U} 238(238 \mathrm{U})$, Th 234 (234 Th), Th 230 (230 Th), Ra 226 (226 Ra), Rn 222 (222 Rn), Po $218\left({ }^{218} \mathrm{Po}\right)$, hasta terminar en el plomo estable.

En al menos 12 de un total de 22 sondeos muestreados en el yacimiento de Marcellus Shale, se han medido, en el agua de retorno, concentraciones de radio $\left({ }^{226} \mathrm{Ra}\right.$ y ${ }^{228} \mathrm{Ra}$ ) de entre 74 y 6540 picoCuries por litro (pCi L-1) (Haluszczak et al., 2012), llegando a máximos de 10000 pCi L-1 (Acharya et al., 2011). El yacimiento de Woodford Shale presenta un rango desde $<20$ hasta $500 \mathrm{pCi} \mathrm{L}^{-1}$ en al agua de retorno
(Acharya et al., 2011). En EE.UU, los niveles umbrales de radio en el agua potable son de $5 \mathrm{pCi} \mathrm{L}^{-1} \mathrm{y}$ de $60 \mathrm{pCi} \mathrm{L}^{-1}$ para efluentes industriales (Rowan et al., 2011). Estos niveles naturales de radiación obligan a los promotores de las explotaciones al tratamiento físico-químico del agua de retorno para alcanzar los niveles exigidos para su eliminación. Una situación parecida podría ocurrir con la radiación presente en los lodos de perforación (Paleontological Research Institution, 2011; Radioactive Waste Material From Tapping Natural Resources: http://www3.epa.gov/ radtown/tapping-natural-resources.html\#learn-more) al alcanzar niveles más elevados que los exigidos para su eliminación en vertedero.

Un primer informe, muy preliminar, del yacimiento de Bowland Shale (U.K) muestra rangos de concentración de radio ( ${ }^{226} \mathrm{Ra}$ ) en agua desde $<0.2$ hasta $90 \mathrm{~Bq} \mathrm{Kg}^{-1}$ (aproximadamente $<5.4$ a 2432 pCi $\mathrm{Kg}^{-1}$ ) (Environment Agency U.K, 2011). En Gran Bretaña el nivel exigido para su eliminación es de $10 \mathrm{~Bq} \mathrm{~g}^{-1}$, aproximadamente $270 \mathrm{pCi} \mathrm{g}^{-1}$ (Radioactive Substances Act, 2011).

Como ya se ha comentado, el radio es el precursor del radón en la serie de trasmutación del uranio y torio. Esta medida de la radiactividad, es la radiactividad natural [Naturally Occurring Radioactive Materials (NORM)] de las pizarras de shale gas, radiactividad que emerge a superficie en el agua de retorno. La concentración de NORM en el agua de retorno no es homogénea en el tiempo, siendo, en un inicio, relativamente baja (debido a que inicialmente existe una mayor proporción de fluido de fracturación que de agua de formación), incrementándose posteriormente a medida que el fluido contiene proporcionalmente mayor concentración de brines (Paleontological Research Institution, 2011).

\section{Constituyentes orgánicos.}

Los componentes orgánicos de un yacimiento de shale gas dependen del tipo de querógeno. En el caso de un yacimiento de querógeno Tipo II aparece metano, etano, propano, n-butano, i-butano y otros hidrocarburos de 5 o más carbonos, e impurezas (mercaptanos, ácido sulfhídrico, nitrógeno, etc). En el caso de un yacimiento de querógeno Tipo III, casi exclusivamente metano (Cohe, 2013). La composición del gas varía de un yacimiento a otro aunque, como término medio, se pueden señalar los valores que aparecen en la Tabla 4, correspondiente a los yacimientos de querógeno Tipo II de Barnett Shale. 


\begin{tabular}{|l|c|}
\hline \multicolumn{1}{|c|}{ Componente } & $\%$ \\
\hline Metano & 85 \\
\hline Etano & 7 \\
\hline Propano & 2 \\
\hline n-butano & 1 \\
\hline i-butano & 1 \\
\hline N.G (C+5) & 0 \\
\hline Nitrógeno & 3 \\
\hline $\mathrm{CO}_{2}$ & 2 \\
\hline Oil & 0 \\
\hline
\end{tabular}

Tabla 4. Porcentajes medios del contenido en componentes orgánicos (Fuente: Cohe, 2013).

Table 4. Mean percentages of the content of organic components (from Cohe, 2013).

\section{Bacterias.}

Entre los componentes vivos presentes en el conjunto materia orgánica-gas se encuentran las bacterias sulfato-reductoras anaerobias de los géneros Desulfovibrio, Desulfomicrobium, Desulfobulbus, Desulfobacter, Desulfobacterium, Desulfococcus, Desulfosarcina, Desulfomonile, Desulfonema, Desulfobotulus y Desulfoarculus (Castro et al., 1999), cuyo ambiente de proliferación es máximo a pH entre 5.5-9. Su metabolismo reduce los sulfatos a $\mathrm{SH}_{2}$ y como ya se ha comentado, este gas puede ascender en disolución en el agua de retorno y de ésta al aire, por lo que para evitar su toxicidad en superficie se inyectan biocidas que eliminan estas bacterias.

\section{B) Aditivos utilizados en la fracturación hidráulica}

Entre los componentes químicos introducidos en la fracturación hidráulica existe una gran diversidad según la función en cada fase del sondeo. Algunos son compuestos químicos inorgánicos, mientras que la mayoría son compuestos químicos orgánicos.

Las funciones de los aditivos son variadas, unas son propias de cualquier sondeo de hidrocarburos convencionales: ácido clorhídrico en disolución, cloruro de potasio, sosa cáustica, bicarbonato sódico, polímeros, etc. Otros son exclusivos de la estimulación por fracturación.

Entre las funciones de estos aditivos se encuentran: ácidos (disuelven los minerales del agua e inician las fracturas en la roca), estabilizadores de arcillas (reducen el hinchamiento de las arcillas y la posibilidad de migrar de éstas, imposibilitando la reducción de la porosidad creada en la fracturación), bactericidas/biocidas (impiden el crecimiento de las bacterias que producen $\mathrm{SH}_{2}$ ), modificadores del $\mathrm{pH}$ (soluciones tampón), inhibidores de la corrosión, crosslinker (mantienen la viscosidad del fluido cuando la temperatura se incrementa), reductores de la viscosidad (disminuyen las pérdidas de carga durante el bombeo), controladores del hierro (impiden la precipitación del hierro disuelto en forma de hidróxidos, carbonatos), disolventes (aditivos solubles usados para controlar la humectabilidad) y surfactantes (reducen la tensión superficial, permitiendo el desplazamiento más libre del agua). Se pueden consultar las listas de productos utilizados en distintos pozos de EE.UU en la siguiente dirección: https://fracfocus.org/chemical-use/what-chemicals-are-used (FracFocus). En ella, además se puede acceder a la ficha de la EPA (Environmental Protection Agency) de un gran número de productos químicos, donde se explican los efectos para la salud debidos a la exposición a dichos productos, entre otros.

\section{Agua de retorno}

El volumen del agua inyectada que retorna a la superficie varía enormemente de unos proyectos a otros y entre operadores. Una estimación de la Agencia de Medio Ambiente de Gran Bretaña establece, como pauta, que una cuarta parte del agua inyectada retorna a la superficie durante semanas hasta algunos meses (Environmental Agency U.K, 2011), entre el 25-75\% según la Agencia de Medio Ambiente de los EE.UU (E.P.A, 2011).

El tiempo que tarda el agua de retorno en volver a la superficie depende, a su vez, del tipo de yacimiento y del operador, siendo la ratio de agua de retorno muy alta los primero días, decreciendo progresivamente, de forma que entre los días 1 a 5 retorna entre el 10-25\%; entre los días 5 a 15 días entre el 8-12\%; entre los días 15 al 30 el $1-5 \%$ y entre los días 30 a 90 el 1-2\% (Acharya et al., 2011).

El agua de retorno en la mayoría de los yacimientos de EE.UU, con querógeno Tipo II, es eminentemente salina, como corresponde al agua donde se formó el gas. Presenta un alto contenido en solidos totales disueltos (TDS), alta demanda biológica de oxígeno (DBO), altas concentraciones de iones $\mathrm{Cl}$, $\mathrm{Br}, \mathrm{Na}, \mathrm{Mg}$ y, en general, pequeñas concentraciones de metales.

En la Tabla 5 se pueden ver distintas concentraciones de elementos mayoritarios del agua de retorno en distintas fechas desde el bombeo en 
Vadillo Fernández, L., et al., 2016. Abordando los retos ambientales del gas de pizarra... Boletín Geológico y Minero, 127 (4): $775-789$

\begin{tabular}{|c|c|c|c|}
\hline Concentración & Unidades & Rango a los 5 días & Rango a los 14 días \\
\hline Sólidos totales en suspensión (TSS) & $\mathrm{mg} \mathrm{L}^{-1}$ & $10.8-3220$ & $17-1150$ \\
\hline Sólidos totales disueltos (TDS) & $\mathrm{mg} \mathrm{L}^{-1}$ & $38500-238000$ & $3010-261000$ \\
\hline Conductividad & $\mu \mathrm{S} \mathrm{cm}^{-1}$ & $79500-470000$ & $6800-710000$ \\
\hline Demanda biológica de oxigeno (DBO) & $\mathrm{mg} \mathrm{L}^{-1}$ & $37.1-1950$ & $2.8-2070$ \\
\hline Dureza (medida como $\mathrm{CaCO}_{3}$ ) & $\mathrm{mg} \mathrm{L}^{-1}$ & $5100-55000$ & $630-95000$ \\
\hline Cloruros & $\mathrm{mg} \mathrm{L}^{-1}$ & $26400-148000$ & $1670-181000$ \\
\hline Bromo & $\mathrm{mg} \mathrm{L}^{-1}$ & $185-1190$ & $15.8-1600$ \\
\hline Sodio & $\mathrm{mg} \mathrm{L}^{-1}$ & $10700-65100$ & $26900-95000$ \\
\hline Sulfatos & $\mathrm{mg} \mathrm{L}^{-1}$ & $2.4-106$ & $<10-89.3$ \\
\hline Hidrocarburos (oil and grease) & $\mathrm{mg} \mathrm{L}^{-1}$ & $4.6-655$ & $<4.6-103$ \\
\hline Radiactividad natural (NORM) & $\mathrm{pCi} \mathrm{L^{-1 }}$ & \multicolumn{2}{|c|}{ ND-18000 (Media 2460) } \\
\hline Bario & $\mathrm{mg} \mathrm{L}^{-1}$ & $21.4-13900$ & $43.9-13600$ \\
\hline Hierro & $\mathrm{mg} \mathrm{L}^{-1}$ & $21.4-180$ & $13.8-242$ \\
\hline
\end{tabular}

Tabla 5. Concentraciones del agua de retorno de sondeos de Marcellus Shale. Fuente: Hammer et al., 2012.

Table 5. Chemical components in water produced from Marcellus Shale Development (from Hammer and VanBriesen, 2012).

el yacimiento de Marcellus Shale (Hammer and VanBriesen, 2012).

El agua de retorno podría ser reutilizada en nuevas fracturaciones hidráulicas. Si bien, la reutilización exige unas condiciones determinadas de salinidad, $\mathrm{Fe}^{2+}$ disuelto, etc., de forma que no generen problemas, como por ejemplo interferencia con las funciones de otros aditivos inyectados, problemas de taponamiento, incrustaciones, etc., requiriendo tecnologías para su adecuación que encarecen el proyecto como pueden ser la osmosis inversa, la evaporación y la destilación termal (Acharya, et al., 2011). Esta problemática da lugar en los EE.UU, en muchas ocasiones, a que se opte por inyectar el agua de retorno en acuíferos profundos (USGS, 2013).

\section{Procesos potenciales de contaminación de acuíferos}

La extensión radial de las fracturas depende del tiempo necesario para que la presión del fluido inyectado pueda romper la roca. La presión del agua y el tiempo durante el que se inyecta agua de manera continua, dependerán de las características del yacimiento, como variaciones litológicas, estado tensional, profundidad, etc. Expresado de otra forma, cuanto más grande es el volumen de agua utilizada más altas serán las presiones y, por otro lado, a mayor rapidez de inyección se generan mayores presiones (The Royal Society and Royal Academy of Engineering, 2012).
Basado en el análisis de monitoreo microsísmico en Marcellus Shale y Barnett Shale los valores máximos de propagación vertical de fracturas registrados fueron de 533 y $588 \mathrm{~m}$, respectivamente (Fisher and Warpinski, 2011).

Osborn et al. (2011) sugieren que la contaminación por metano encontrada en acuíferos asociados a los yacimientos de Utica y Marcellus Shale (Pensilvania) podría haberse producido bien a través de las fisuras laterales y verticales que se producen en el cemento del pozo o del acero deteriorado de antiguos sondeos abandonados o, con menor probabilidad, a través de las fracturas y fallas conectadas mediante la fracturación hidráulica, así como. En este mismo artículo, Osborn et al., (2011) presentan los resultados del análisis del contenido en metano que aparece en pozos de agua potable, próximos a sondeos de inyección. Con el fin de identificar la procedencia del metano, el estudio diferencia el metano profundo (termogénico) del metano superficial (biogénico) por la desviación del isótopo del carbono $13\left(\delta^{13} \mathrm{C}\right)$. Estos pozos experimentaron un incremento de metano termogénico de entre 19.2 y $64 \mathrm{mg} \mathrm{L}^{-1}$, excediendo la solubilidad del metano en agua y con riesgo de explosiones de metano.

Se desconoce cuál es el verdadero mecanismo de paso del metano, existiendo diversas posibilidades: a través de las fisuras del cemento de antiguos sondeos abandonados, a través de las fisuras abiertas en el cemento en el sondeo debido a las vibraciones de la fracturación hidráulica, lentamente a través de 
fallas abiertas, o a través de fallas cerradas que habrían sufrido un deslizamiento de falla.

The Royal Society and The Royal Academy of Engineering considera que la migración de gas a través de fallas abiertas es posible en yacimientos convencionales pero no en pizarras ya que, dada su baja permeabilidad, el gas no puede fluir sin condiciones de presión adecuadas y que la fracturación se realiza con este condicionante. Incluso, en el caso de fallas cerradas, pequeños sismos de magnitud local de 2.3, como el de Blackpool (UK), debido a la sismicidad inducida por la fracturación, podrían haber dado lugar a pequeños deslizamientos de falla al aumentar la presión de poro de los materiales de la misma. El deslizamiento de falla podría haber sido de $1 \mathrm{~cm}$ en una falla de $70^{\circ}$ de buzamiento (The Royal Society \& Royal Academy of Engineering, 2012). La posibilidad de una fuga de fluido a través del plano de falla era muy baja, en el peor de los casos esta fuga estaría limitada por los materiales impermeables que yacen encima del yacimiento de Bowland Shale (Green et al., 2012). El seísmo dio lugar una deformación del casing de más de 0.5 pulgadas (Pater and Baisch, 2011).

La complejidad del problema es de tal envergadura que la Agencia de Medio Ambiente Americana ha lanzado un proyecto para modelizar diversos escenarios de posibles fugas de metano. El modelo desarrolla diversos escenarios de posibles mecanismos de fugas de fluidos, incluido gas, a acuíferos superficiales: Caso A, paso de fluidos a través de fisuras en el cemento de revestimiento del sondeo dañado por la fracturación; Caso B1, paso de fluidos a un acuífero subsuperficial a través de una fractura inducida; Caso B2, semejante al anterior pero la fractura inducida afecta previamente a otro yacimiento de gas localizado por encima del yacimiento de shale gas; Caso $C$, paso a través de una falla sellada y activada por la fracturación inducida; Caso D1, paso de fluidos a través del cemento fisurado de otros sondeos antiguos; Caso D2, paso de fluidos a través de la rotura del acero del sondeo de sondeos antiguos (E.P.A., 2012; Reagan et al., 2015). En su estudio Hydraulic Fracturing Drinking Water Assessment (E.P.A., 2015), la EPA concluye que no se han encontrado evidencias de que ninguno de estos mecanismos se produzca de forma sistemática o extendida en los Estados Unidos, y que el número de casos de contaminación acontecidos es pequeño en relación al número de pozos abiertos.

Como medidas de prevención y basándose en estudios de máxima propagación de fracturas se comienzan a proponer determinadas distancias de seguridad ante la posible contaminación de acuíferos.
Por ejemplo, el gobierno de Quebec (Canadá), con fecha de 29 de mayo de 2013, publicó un proyecto que reglamenta una distancia mínima de 400 metros de cualquier segmento de fracturación con respecto a la base de un acuífero (Gazzete Officielle du Quebec Partie 2, N²2, 29 Mai 2013).

La tendencia general en Estados Unidos (dependiendo del Estado) para evitar posibles fugas de fluidos, es que los operadores mantengan un control de la integridad del pozo, mediante pruebas que garanticen la integridad de las tuberías, el aislamiento de la cementación y el control de la adherencia tubería-cemento-formación (registros CBL) de todo el pozo. Dichos controles deben certificar que no se han formado fisuras en el pozo y que no existe comunicación entre las tuberías, cementaciones y los terrenos perforados. Estos estudios deben ser realizados por una entidad independiente, enviando los registros de presión correspondientes al Ente Regulador. Dichos controles de presión deben repetirse ante cualquier evento que modifique las condiciones primarias naturales del medio físico y que puedan alterar la integridad de los pozos (American Petroleum Institute, 2009; Bolander, 2011).

\section{Recuperación de metano}

En un yacimiento de gas convencional el factor de recuperación oscila entre $70-90 \%$ según Muggeridge et al. (Muggeridge et al., 2014) o entre $50-80 \%$ según Jahn, et al. (Jahn, et al., 2008). En el caso de un yacimiento no convencional, el factor de recuperación varía entre desde menos del $15 \%$ hasta el $30 \%$, considerándose factores de recuperación del $30 \%$ como excepcionales (U.S. Energy Information Administration, 2013). Sandrea and Sandrea (2014) consideran que el factor de recuperación de los principales yacimientos americanos serían: $1.7 \%$ en Haynesville; $6.1 \%$ en Barnett, $11.2 \%$ en Fayetteville y $9.3 \%$ en Marcellus.

El factor de recuperación del gas depende de diversos factores como el tamaño de poro, la presión hidrostática de la formación, el contenido en arcilla y la complejidad tectónica. Las formaciones con alto contenido en arcilla tienden a deformarse en lugar de romperse, dando lugar relativamente a un pequeño número de fracturas inducidas. La presencia de saltos de falla limitan el desarrollo de la perforación dirigida, fallas con tectónica compresiva reducen la permeabilidad de la formación y su capacidad de flujo (U.S. Energy Information Administration. 2013).

Un bajo factor de recuperación unido a la perdida de presión de la formación, da lugar a una alta 
declinación del caudal de los pozos, lo que obliga a las operadoras a una constante apertura de nuevos pozos en los yacimientos de EE.UU., bien por falta de rentabilidad o por la necesidad de cumplir el contrato de suministro de gas con las empresas de los gasoductos.

En el yacimiento de Haynesville el caudal de los pozos disminuye un $68 \%$ el primer año, llegando al cuarto año a una disminución del caudal de un $96 \%$, habiéndose abierto unos 3000 pozos productivos en el periodo que va del 2008 al 2012; en Marcellus la disminución el cuarto año es del 97\%, habiéndose abierto unos 4000 pozos productivos en el periodo 2006-2011; en Barnett la declinación es de un $61 \%$ el primer año, llegando al quinto año a una disminución del caudal de un $87 \%$, habiéndose abierto 12000 pozos en el periodo 2006-2012 (Hughes, 2013).

Los pozos abandonados o inactivos debido a la declinación de la producción, suponen una fuente potencial de contaminación de acuíferos. En Estados Unidos, las regulaciones estatales y federales están poniendo el foco en la distribución y condición de aquellos pozos que penetran acuíferos y que se encuentran abandonados (Texas Railroad Commission, 2000; U.S.G.S., 2013; Abandoned \& Orphaned Well Program, Pennsylvania), estableciendo directrices para la clausura de los pozos y el seguimiento tras el abandono.

\section{Ocupación de suelo}

El emplazamiento donde se aloja la torre de perforación y el resto de equipamiento recibe, en la terminología anglosajona de operadores de hidrocarburos, el nombre well-pad. En este emplazamiento se instalan, además de la torre de perforación, la balsa de lodos, balsa de agua de inyección, balsas de agua de retorno o flow-back, diversos equipos auxiliares (camiones, compresores, condensadores, deshidratadores, contenedores de aditivos, etc), además de pistas y gasoductos de conexión a la red de distribución principal. Todo el equipo se retira una vez realizada la operación de fracturación hidráulica.

Desde hace escasos años se están realizando varios sondeos por plataforma (multi-well pad), de forma que en cada plataforma se pueden realizar entre 4 y 18 sondeos, separados por 5-6 metros de distancia, con un sondeo horizontal por cada sondeo vertical. Este sistema permite reducir la ocupación del suelo, la apertura de pistas, la instalación de gasoductos, los permisos necesarios, el tráfico de camiones, etc., ya que es necesaria una única torre de perforación para la realización de varios sondeos con desplazamientos de pocos metros (5-6 $\mathrm{m}$ ), con el consiguiente ahorro de tiempo, aumento de la eficacia en la producción debido a la concentración de equipos de operación y vigilancia, mejora del área de drenaje del campo de gas y, por supuesto, ahorro de costes debido a la economía de escala (Dawson et al., 2012).

La superficie ocupada por cada plataforma (well pad) es aproximadamente de $16000-20000 \mathrm{~m}^{2}$, aumentando ligeramente por cada sondeo añadido (multi-well pad) (U.S. Department of Energy, 2009). Una plataforma de seis a ocho sondeos podría ocupar una superficie de $30000 \mathrm{~m}^{2}$ (New York Department of Environmental Conservation, 2011). Esta superficie ocupada disminuye una vez acabada la fase de fracturación hidráulica, quedando reducida a una superficie menor con los cabezales de los pozos durante la fase de operación de extracción del gas.

La densidad de sondeos por superficie varía de un yacimiento a otro, así, en el yacimiento de Barnett Shale Play (Texas) la densidad de sondeos es de 5.5 sondeos por milla cuadrada, en Haynesville Shale Play (Luisiana), Fayetteville Shale Play (Arkansas) y en Marcellus Shale Play (Pensilvania, New York, Ohio, etc.), la densidad es de 8 pozos por milla cuadrada (U.S. Energy Information Administration, 2011).

Esta alta densidad de sondeos por superficie es consecuencia de la alta declinación de los pozos, lo que obliga a las operadoras a abrir continuamente nuevos pozos.

\section{Sismicidad inducida}

Sin duda alguna es en la extracción de gas "no convencional" mediante fracturación hidráulica con agua a presión donde más se ha podido comprobar la generación de sismicidad inducida gracias a la monitorización de fracturas, in situ, de muchos de estos yacimientos. Uno de los que tuvo más repercusión mediática fue el yacimiento de shale gas de Bowland, en Blackpool (Gran Bretaña) donde, entre el 31 de marzo y el 27 de mayo de 2011, se detectaron 50 seísmos en un área no sísmica, durante la fase de fracturación hidráulica. El mayor $M_{L} 2.3$ se produjo el 1 de abril cuando la presión en el fondo del sondeo alcanzaba, aproximadamente, 9900 psi, 68.2 MPa (Eisner, et al., 2011). Presión necesaria para abrir los planos de fractura de la pizarra con un alto gradiente de esfuerzos horizontales, mínimo de $0.75 \mathrm{psi} / \mathrm{ft}$ y máximo de $1.25 \mathrm{psi} / \mathrm{ft}$. Este seísmo dio lugar a la paralización del sondeo de investigación. Posteriormente se comprobó que el tubo de acero (casing) del sondeo se había deformado en más de 0.5 pulgadas a una profundidad entre 8480 y 8640 pies (2584 y 2633 m, respectivamente) (Pater and Baisch, 2011). 
Green et al. (2012) sugieren dos posibles teorías: la primera que el sondeo inyectara directamente el agua a presión en la misma falla; la segunda que el sondeo inyectará agua a unos pocos cientos de metros de la falla, decantándose por la primera de ellas. La falla tiene una orientación $50^{\circ} \mathrm{N}$, buzamiento $70^{\circ}$ W y podría haber producido un deslizamiento de falla de $1 \mathrm{~cm}$ y un área de ruptura de $10000 \mathrm{~m}^{2}$.

Para prevenir la sismicidad inducida relevante (The Royal Society and Royal Academy of Engineering, 2012) propuso un sistema de vigilancia de tipo semáforo. Se estima que un sistema de tipo semáforo, ligado al monitoreo de la actividad sísmica en tiempo real, es una estrategia de mitigación esencial. Sin embargo, esto requiere la definición de unos valores umbral de magnitudes aceptables para el cese y el comienzo de las operaciones.

En el caso de la cuenca de Bowland, el valor propuesto inicialmente para el cese de las operaciones fue 1.7 $\mathrm{M}_{\mathrm{L}}$ (Pater and Baisch, 2011). Dicho valor se basa en la magnitud crítica de $2.6 \mathrm{M}_{\mathrm{L}}$ y un máximo incremento post-inyección de la magnitud estimado en $0.9 \mathrm{M}_{\mathrm{L}}$. Se adoptó el valor crítico de $2.6 \mathrm{M}_{\mathrm{L}}$ como límite permitido, basándose en la normativa alemana para vibraciones del terreno, asegurando de esta manera que no se puedan generar daños en las estructuras de superficie cercanas al pozo donde se esté realizando la estimulación. Se establecieron entonces los siguientes umbrales:

- $M_{L}<0$ : Proceder con las operaciones regulares.

- $0<\mathrm{M}_{\mathrm{L}} \leq 1.7$ : Continuar con la monitorización tras la inyección durante dos días al menos, hasta que la tasa de sismicidad haya caído por debajo de un evento al día.

- $M_{L}$ >1.7: Detener la inyección y emplear el flujo de retorno para reducir la presión, mientras continúa la monitorización.

No obstante, Green et al. (2012), señalan que con estos valores no se hubiera podido adoptar ninguna medida de mitigación en el caso del evento de magnitud 2.3 $\mathrm{M}_{\mathrm{L}}$ en Preese Hall. Por esta razón, los autores proponen un valor umbral de $0.5 \mathrm{M}_{\mathrm{L}}$ para el cese de las operaciones.

\section{Conclusiones}

La fracturación hidráulica para la obtención de gas no convencional puede presentar impactos ambientales que deben considerarse a la hora de la toma de decisiones por parte de las autoridades ambientales. En España, de mayor a menor incidencia ambiental se encontrarían:
La pérdida de calidad ambiental de la superficie del yacimiento debido al alto número de sondeos a perforar, perdida de usos agrícolas, ganaderos, etc. Esta alta ocupación del suelo se podría mitigar mediante el desarrollo de varios sondeos por pad ( $\mathrm{mul}$ ti-well pad).

El consumo de agua por yacimiento puede ser relativamente alto (entre 10000 y $15000 \mathrm{~m}^{3}$ por sondeo), pudiendo entrar en conflicto con otros usos del agua en la zona afectada. Asimismo, un incremento de las extracciones de aguas subterráneas en un área, puede tener afecciones sobre las masas de agua superficiales dependientes del acuífero explotado, afección a caudales ecológicos de ríos, secado de manantiales. El uso de recursos superficiales genera conflictos con usuarios situados aguas abajo de las tomas y la afección a los caudales ecológicos. Una posibilidad es buscar fuentes alternativas al agua de inyección proveniente de ríos y acuíferos, como por ejemplo la utilización de aguas residuales regeneradas.

El agua de retorno tiene una alta concentración salina, por lo que para poder reutilizarla hay que rebajar estas concentraciones mediante tecnologías de osmosis inversa y evaporación/destilación termal, lo que genera una salmuera residual. La eliminación de dicha salmuera mediante su inyección en formaciones geológicas, precisa una investigación hidrogeológica muy precisa y costosa, ya que se necesita un acuífero receptor del efluente, aislado del funcionamiento hidrogeológico regional. Además, en el marco legal español, en relación con el vertido de aguas residuales susceptibles de afectar la calidad de las aguas subterráneas, precisa de una autorización administrativa. En el caso de inyecciones de aguas residuales en acuíferos, éstas están prohibidas salvo si se demuestra que el acuífero receptor tiene una calidad tal, que no es posible su uso. En el caso de vertidos indirectos, la autorización solo se podría obtener si un estudio hidrogeológico demostrase que el vertido es inocuo para la calidad de las aguas subterráneas.

Existe la posibilidad de que ocurran fugas de metano hacia acuíferos superficiales, principalmente, a través de las fisuras del cemento del sondeo. Es necesario un control de pruebas de integridad del sondeo, cementaciones; evitar la proximidad a pozos antiguos, etc.

La misma Comisión Europea, propone la recomendación de someter a Evaluación Ambiental Estratégica para prevenir, gestionar y reducir los impactos y los riesgos para la salud humana y el medio ambiente (Recomendación de la Comisión de 22 de enero de 2014, relativa a unos principios mínimos para la exploración y producción de hidrocarburos (como el gas de esquisto) utilizando la fracturación 
Vadillo Fernández, L., et al., 2016. Abordando los retos ambientales del gas de pizarra... Boletín Geológico y Minero, 127 (4): $775-789$

hidráulica de alto volumen, de Planes y Programas de fracturación hidráulica.

\section{Referencias}

Abandoned \& Orphaned Well Program, Pennsylvania. Office ofOiland Gas Management.DepartmentofEnvironmental Protection. Pennsylvania. 10/06/2015. http://www. portal.state.pa.us/portal/server.pt/community/ abandoned__orphan_well_program/20292.

Acharya, H.R., Henderson, C., Matis, H., Kommepalli, H., Moore, B. and Wang, H. 2011. Cost Effective Recovery of Low-TDS Frac Flowback Water for Re-use. U.S. Department of Energy, Washington D.C, 100 pp.

American Petroleum Institute. 2009. Hydraulic fracturing operations: well construction and integrity guidelines. API guidance document HF1. American Petroleum Institute. Washington DC, $24 \mathrm{pp}$.

American Petroleum Institute. 2010. Water Management Associated with Hydraulic Fracturing. API guidance document HF2. American Petroleum Institute. Washington DC, $26 \mathrm{pp}$.

Beard, T. 2011. Fracture Design in Horizontal Shale Wells - Data Gathering to Implementation. EPA Hydraulic Fracturing Workshop, March 10th - 11th, 2011. http:// water.epa.gov/type/groundwater/uic/class2/hydraulicfracturing/upload/fracturedesigninhorizontalshalewells.pdf.

Behar, F., Beaumont, V. and Penteado, B. 2001. Rock-Eval 6 Technology: Performances and Developments. Oil \& Gas Science and Technology - Rev. IFP., 56 (2), 111-134.

Bolander, J. 2011. Assessment Methods for Well Integrity During the Hydraulic Fracturing Cycle, March 11, 2011. EPA's Study of Hydraulic Fracturing and Its Potential Impact on Drinking Water Resources. Technical Presentation Session 8: Pre- \& Post-Hydraulic Fracturing Well Integrity. 21pp.

Boyer, C., Kieschnick, J., Suarez-Rivera, R. and Lewis, R.E. 2006. Producing gas from its source. Oilfield Review. 18 (3), 36-49.

Bruner, K.R. and Smosna, R. A. 2011. A Comparative Study of the Mississippian Barnett Shale, Fort Worth Basin, and Devonian Marcellus Shale, Appalachian Basin. National Energy Technology Laboratory. U.S. Department of Energy, Washington D.C, USA, 106 pp.

Castro, H.F., Wiliamns, N.H. and Ogram, A. 1999. Phylogeny of sulfate-reducing bacteria. FEMS Microbiology Ecology, 31, 1-9.

Cohe, A.K. 2013. The Shale Gas Paradox: Assessing the Impacts of the Shale Gas Revolution on Electricity Markets and Climate Change. Harvard Kennedy School, $135 \mathrm{pp}$.

Consejo Superior de Colegios de Ingenieros de Minas. 2013. Gas no convencional en España, una oportunidad de futuro. Consejo Superior de Colegios de Ingenieros de Minas, $149 \mathrm{pp}$.

Dawson, M., Howard, P., Salkeld, M. 2012. Improved Productivity in the Development of Unconventional
Gas. Productivity Alberta Collaboration, Innovation and Transformation, $61 \mathrm{pp}$.

EIA/ARI. 2013. World Shale Gas and Shale Oil Resource Assessment Technically Recoverable Shale Gas and Shale Oil Resources: An Assessment of 137 Shale Formations in 41 Countries Outside the United States. U.S. Energy Information Administration and U.S. Department of Energy. Advanced Resources International, Inc. 707 pp.

Eisner, L., Janska, E. and Matousek, P. 2011. Seismic Analysis of the events in the vicinity of the Preese Hall well. Seismik final report for Cuadrilla Resources Ltd. $28 \mathrm{pp}$.

Environment Agency U.K. 2011. North west-monitoring of flowback water, U.K, 8 pp.

E.P.A. 2011. Draft Plan to Study the Potential Impacts of Hydraulic Fracturing on Drinking Water Resources. U.S. Environmental Protection Agency. Washington D.C, USA, 126 pp.

E.P.A. 2012. Study of the Potential Impacts of Hydraulic Fracturing on Drinking Water Resources. U.S. Environmental Protection Agency. Washington, $262 \mathrm{pp}$.

E.P.A. 2015. Assessment of the Potential Impacts of Hydraulic Fracturing for Oil and Gas on Drinking Water Resources. U.S. Environmental Protection Agency, Washington, DC, EPA/600/R-15/047, 998 pp.

Fisher, K. and Warpinski, N. 2011. Hydraulic-FractureHeight Growth: Real Data. SPE 145949, 18 pp.

FracFocus. FracFocus Chemical Disclosure Registry. Ground Water Protection Council and Interstate Oil and Gas Compact Commission. 12/01/2015. https://fracfocus.org/chemical-use/what-chemicals-are-used.

Green, A., Styles, P. and Baptie, B.J. 2012. Preese Hall Shale gas fracturing review \& recommendations for induced seismic mitigation. Department of Energy and Climate Change, $26 \mathrm{pp}$.

Haluszczak, L.O., Rose, A.W. and Kump, L.R. 2012. Geochemical evaluation of flowback brine from Marcellus gas wells in Pennsylvania, USA. Applied Geochemistry, 28, 55-61.

Hammer, R. and VanBriesen, J. 2012. In Fracking's Wake: New Rules are Needed to Protect Our Health and Environment from Contaminated Wastewater. Natural Resources Defense Council, 31 pp.

House of Commons. Energy and Climate Change Committee. 2011. Shale Gas. Fifth Report of Session 2010-2012. Volume I, 43 pp.

Hughes, J.D. 2013. Drill, Baby, drill. Can Unconventional Fuels Usher in a New Era of Energy Abundance? Post Carbon Institute, California, U.S.A, 166 pp.

Jahn, F., Cook, M. and Graham, M. 2008. Hydrocarbons Exploration and Production. 2 ${ }^{\text {ND }}$ Edition. Developments in Petroleum Science. Eselvier, $444 \mathrm{pp}$.

Leventhal, J.S., Crock, J.C. and Malcom, M.J. 1981. Geochemistry of trace elements and uranium in Devonian shales of the Appalachian Basin. United States Department Geological Survey, 73 pp.

McCarty, K., Rojas, K., Niemann, M., Palmowski, D., Peters, K. and Stankiewicz, A. 2011. Basic Petroleum 
Geochemistry four Source Rock Evaluation. Oilfield Review. 23 (2): 32-43.

MINETUR. Ministerio de Industria, Energía y Turismo. Mapa de posición de permisos de investigación y concesiones de explotación y almacenamiento subterráneo de hidrocarburos. 10/06/2015. http://www6.mityc. es/aplicaciones/energia/hidrocarburos/petroleo/exploracion2013/mapas/inicio.html.

Muggeridge, A., Cockin, A., Webb, K., Frampton, H., Collins, I., Moulds, T. and Salino, P. 2014. Recovery rates, enhanced oil recovery and technological limits. Philosophical Transactions of The Royal Society A, 372: 20120320.

New York State Department of Environmental Conservation. 2011. Revised Draft Supplemental Generic Environmental Impact Statement. On The Oil, Gas and Solution Mining Regulatory Program. New York State Department of Environmental Conservation, $1236 \mathrm{pp}$.

Osborn, S.G., Vengosh, A., Warner, N.R. and Jackson, R.B. 2011. Methane contamination of drinking water accompanying gas-well drilling and hydraulic fracturing. Proceedings of the National Academy of Sciences, 108: 8172-8176.

Paleontological Research Institution. 2011. Understanding Naturally Occurring Radioactive Material in the Marcellus Shale. The Marcellus Shale: The Science Beneath the Surface, Issue Number 4.8 pp.

Pater, C.J. and Baisch, S. 2011. Geomechanical study of Bowland shale seismicity. Synthesis report, $57 \mathrm{pp}$.

Pickett, A. 2009. New solutions emerging to treat and recycle water used in hydraulic fracs. American Oil \& Gas Reporter.

Radioactive Substances Act 1993. 2011. The Environmental Permitting (England and Wales) (Amendment) Regulations. Guidance for NORM industrial activities on how to comply with the radioactive substances exemption regime, $10 \mathrm{pp}$.

Radioactive Waste Material From Tapping Natural Resources. EPA. 10/06/2015. http://www3.epa.gov/radtown/tapping-natural-resources.html\#learn-more.

Reagan, M.T., Moridis, G.J., Johnson, J.N. and Keen, N.D. 2015. Numerical simulation of the environmental impact of hydraulic fracturing of tight/shale gas reservoirs on near-surface groundwater: background, base cases, shallow reservoirs, short-term gas and water transport. Water Resources Research, 51, 1-31.

Rowan, E.L., Engle, M.A., Kirby, C.S. and Kraemer, T.F. 2011. Radium content of oil and gas fields produced waters in the Northern Appalachian Basin (USA): Summary and discussion of Data. United States Geological Survey (U.S.G.S.), $31 \mathrm{pp}$.

Sandrea, R. and Sandrea, I. 2014. New Well- Productivity Data Provides US Shale Potential Insights. Oil and Gas Journal, 112: 66-77.

Texas Railroad Commission. 2000. Well plugging primer. Oil and Gas Division - Well Plugging Section. 20 pp. 10/06/2015. http://www.rrc.state.tx.us/media/6358/plugprimer1.pdf

The Royal Society and Royal Academy of Engineering. 2012. Shale gas extraction in the UK: a review of hydraulic fracturing. $75 \mathrm{pp}$.

TOTAL. Total Commited to Better Energy. New applications for proven techniques. 10/06/2015. http://www. total.com/en/energies-expertise/oil-gas/exploration-production/strategic-sectors/unconventional-gas/ presentation/new-applications-proven-techniques.

U.S. Energy Information Administration. 2011. Review of Emerging Resources: U.S. Shale Gas and Shale Oil Plays. Washington, $82 \mathrm{pp}$.

U.S. Energy Information Administration. 2013. Technically Recoverable Shale Oil and Shale Gas Resources: An Assessment of 137 Shale Formations in 41 Countries outside the United States, Washington, 20 pp.

U.S.G.S. 2013. Water resources and shale gas/oil production in the Appalachian Basin - Critical Issues and Evolving Developments. Open-File Report 2013-1137. 12 pp.

U.S. Department of Energy. Office of Fossil Energy National Energy Technology Laboratory. 2009. Modern Shale Gas. Development in the United States: A Primer. U.S. Department of Energy. Office of Fossil Energy National Energy Technology Laboratory., U.S. Department of Energy, Washington D.C, USA, 98 pp.

Veil, J. A. 2010. Water management technologies used by Marcellus Shale gas producers. Final Report. US Department of Energy, Office of Fossil Energy, National Energy Technology Laboratory. $59 \mathrm{pp}$.

Recibido: febrero 2015

Revisado: junio 2015

Aceptado: junio 2015

Publicado: diciembre 2016 
